\title{
ON THE EXISTENCE OF SEQUENCES OF CO-PRIME PAIRS OF INTEGERS
}

\author{
DAVID L. DOWE
}

(Received 23 November 1987)

Communicated by J. H. Loxton

\begin{abstract}
We say that a positive integer $d$ has property (A) if for all positive integers $m$ there is an integer $x$, depending on $m$, such that, setting $n=m+d, x$ lies between $m$ and $n$ and $x$ is co-prime to $m n$. We show that infinitely many even $d$ and infinitely many odd $d$ have property (A) and that infinitely many even $d$ do not have property (A). We conjecture and provide supporting evidence that all odd $d$ have property (A).

Following A. R. Woods [3] we then describe conditions $\left(A_{u}\right)$ (for each $u$ ) asserting, for a given $d$, the existence of a chain of at most $u+2$ integers, each co-prime to its neighbours, which start with $m$ and increase, finishing at $n=m+d$. Property (A) is equivalent to condition $\left(A_{1}\right)$, and it is easily shown that property $\left(A_{i}\right)$ implies property $\left(A_{i+1}\right)$. Woods showed that for some $u$ all $d$ have property $\left(\mathrm{A}_{u}\right)$, and we conjecture and provide supporting evidence that the least such $u$ is 2 .
\end{abstract}

1980 Mathematics subject classification (Amer. Math. Soc.) (1985 Revision): 11 A 05.

In [3] Woods proved that there is a constant $L$ such that if $m, n$ are positive integers with $d=n-m>L$, then there is a sequence of numbers $m<x_{1}<$ $x_{2}<\cdots<x_{l}<n$ with $1 \leq l \leq L$ having greatest common divisors satisfying $\left(m, x_{1}\right)=1,\left(x_{i}, x_{i+1}\right)=1$ for $1 \leq i<l,\left(x_{l}, n\right)=1$. This led Woods to conjecture that $L=1$, that is, to conjecture that all numbers $d>1$ have

Property (A). For all natural numbers $m, n$ with $n-m=d$ there is some $x$ with $m<x<n$ and $(x, m n)=1$.

(C) 1989 Australian Mathematical Society $0263-6115 / 89 \$ A 2.00+0.00$ 
However, as Woods (private communication) has observed, this conjecture is false, the smallest counterexample being $d=16, m=2184=2^{3} .3 .7 .13$, $n=2200=2^{3} \cdot 5^{2} \cdot 11$. This immediately gives infinitely many counterexamples, as we now show. Since $m<x<n$ implies $(x, m)<d$ and $(x, n)<d$, it follows that if $(x, m n)>1$ then $p \mid(x, m n)$ for some prime $p<d$. Thus if $m=m_{0}, n=n_{0}$ is a counterexample to $d$ having property (A) and $P$ is the product of all prime numbers less than $d$, then $m=m_{0}+t P, n=n_{0}+t P$ gives another such counterexample for each natural number $t$.

It is thus natural to ask which values of $d$ have property (A).

We answer this question for numbers $d$ of certain forms, from which we show that property (A) holds for infinitely many even $d$ (and for infinitely many odd $d$ ) and fails for infinitely many even $d$. We also modify the (incorrect) original conjecture to

Conjecture 1. All odd $d>1$ have property (A); that is, if $n-m>1$ is odd, then there is some $x$ with $m<x<n$ and $(x, m n)=1$.

Note. The author has proved this conjecture for all odd $d \leq 89$ and believes it to be true for all odd $d \leq 219$. A referee has checked the validity of the conjecture for $1 \leq m \leq 1000, d=3,5, \ldots, 501$.

TheOREM 1. Let $t>1$. Let $q_{1}>2, q_{2}>q_{3}>\cdots>q_{t}>2$ be primes, $1 \leq i \leq t$. If $d<q_{1}^{t}, d<q_{t} \min \left(q_{1}, q_{t}\right), q_{2}=d-q_{1}, q_{3}=d-q_{1}^{2}, \ldots, q_{t}=$ $d-q_{1}^{t-1}$ and $d \equiv 1 \bmod q_{i}$, then $d$ does not have property (A). Furthermore, $a$ specific $m$ and $n$ illustrating the counterexample can be obtained by requiring that $q_{1} q_{2} \cdots q_{t} \mid n$ and that all other primes less than $d$ divide $m$.

Proof. Initially requiring that all primes less than $d$ divide $m$ takes care of all numbers between $m$ and $n$ except $x=m+1$. Now, if we no longer require that $q_{1} \mid m$, nor that $q_{2} \mid m, \ldots$, nor that $q_{t} \mid m$, then the only numbers between $m$ and $n=m+d$ still requiring attention will be $m+1, m+q_{1}, \ldots, m+q_{1}^{t-1}$, $m+q_{2}, \ldots, m+q_{t-1}$ and $m+q_{t}$; that is, $n-(d-1), n-q_{2}, \ldots, n-q_{t}$, $n-q_{1}, \ldots, n-q_{1}^{t-2}$ and $n-q_{1}^{t-1}$. The requirement that $q_{1} q_{2} \cdots q_{t} \mid n$ takes care of all of these since $d-1 \equiv 0 \bmod q_{i}$.

Theorem 1 gives us a method for producing $d$ not satisfying property (A).

EXAMPLE 1: with $t=2, i=1$ and so $q_{1}<q_{2}$.

$q_{1}=5 ; q_{2}=11$. This gives 2.3.7.13|m, 2.5.11 $\mid n=m+d=m+5+$ $11=m+16$ and we have seen this one before.

$q_{1}=7 ; q_{2}=29$.

$q_{1}=11 ; q_{2}=23,67,89$.

Etc. 
EXAMPLE 2: with $t=3$ and $i=1$.

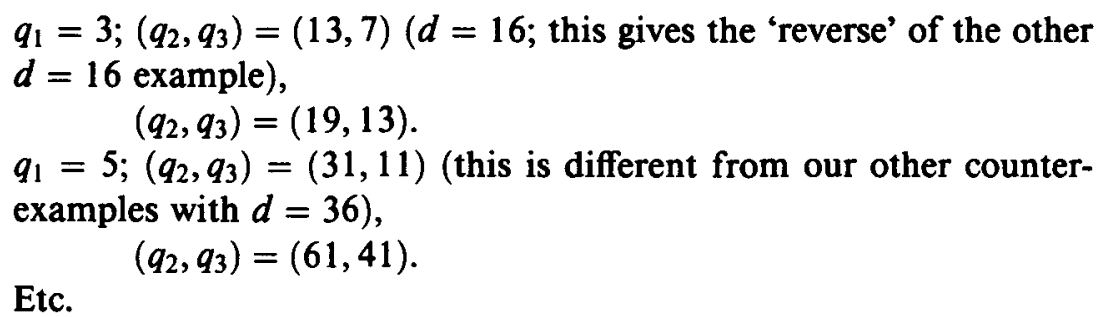

As we might suspect from the examples, property (A) fails for infinitely many even values of $d$.

Let $P(k, l)$ be the least prime in the arithmetic progression $n \equiv l(\bmod k)$, where $\operatorname{gcd}(k, l)=1$.

LEMMA 2 [2]. Given $\varepsilon>0$, there exists a constant $c(\varepsilon)$ and infinitely many primes $q$ such that $P(q, 1)<c(\varepsilon) q^{\theta+\varepsilon}$, where $\theta=2 e^{1 / 4}\left(2 e^{1 / 4}-1\right)^{-1}=$ $1.63773 \ldots$

COROLlARY 3. There exist infinitely many pairs of primes $p, q$ satisfying $p \equiv 1 \bmod q$ and $p<q^{2}-q$.

It follows from Theorem 1 (with $t=2$ and $i=1$ ) and Corollary 3 that property (A) fails for infinitely many even values of $d$.

It turns out that property (A) holds for infinitely many even values of $d$ (and infinitely many odd values of $d$ ).

THEOREM 4. If either

(a) $d=q^{\gamma}+1, q$ a prime, $\gamma \geq 0$,

or

(b) $d=p_{1}^{\beta_{1}}+p_{2}^{\beta_{2}}=p_{1}^{\alpha_{1}} p_{2}^{\alpha_{2}}+1$, where $p_{1}, p_{2}$ are distinct primes, $\beta_{1}, \beta_{2}, \alpha_{1}$, $\alpha_{2}>0$,

then $d$ has property (A).

Proof. (a) Let $d=q^{\gamma}+1$. If $\gamma=0$, we can take $x=m+1$. If $\gamma>0$, then if $q \nmid n$ we can take $x=m+1$, while if $q \nmid m$ we can take $x=n-1$.

(b) If $p_{1} \nmid m$ and $p_{2} \nmid n$, we can take $x=m+p_{1}^{\beta_{1}}$. Similarly, if $p_{2} \nmid m$ and $p_{1} \nmid n$, we can take $x=m+p_{2}^{\beta_{2}}$. Finally, if $p_{1} p_{2} \mid m$ we can take $x=m+1$; while if $p_{1} p_{2} \mid n$, then $x=n-1$ suffices.

It follows from Case (a) of Theorem 4 with $q$ an odd prime that there are infinitely many even values of $d$ with property (A); and with $q=2$ it follows that there are infinitely many odd values of $d$ with property (A). 
Between them, Theorems 1 and 4 go some way toward classifying all values of $d$. The cases unclassified by Theorems 1 and 4 for $d \leq 38$ are $d=$ $11,23,27,29,31,35,37$. These can be all shown to have property (A).

We note that Theorems 1 and 4 classified all even values of $d \leq 38$.

Question. Do Theorems 1 and 4 classify all even values of $d$ ?

As we mentioned at the start of the paper, Woods [3] proved that there is a constant $L$ such that if $m, n$ are positive integers with $d=n-m>L$, then there is a sequence of numbers $m<x_{1}<\cdots<x_{l}<n$ with $1 \leq l \leq L$ having greatest common divisors satisfying $\left(m, x_{1}\right)=1,\left(x_{i}, x_{i+1}\right)=1$ for $1 \leq i<l$, $\left(x_{l}, n\right)=1$. We have shown that the smallest such $L$ is at least 2 ; we now try to find it.

First, we generalize the notion of property (A).

Definitions. Say $x \prec y$ if and only if $\operatorname{gcd}(x, y)=1$ and $x<y$.

Say $x \preccurlyeq y$ if and only if $(\operatorname{gcd}(x, y)=1$ and $x<y)$ or $x=y$.

Definition. For each $u \in \mathbf{N}$ we say that $d>1$ has property $\left(\mathrm{A}_{u}\right)$ if and only if

$$
\forall m \forall n\left(m<n=m+d \rightarrow \exists z_{1}, z_{2}, \ldots, z_{u}, m \preccurlyeq z_{1} \preccurlyeq z_{2} \preccurlyeq \cdots \preccurlyeq z_{u} \preccurlyeq n\right) .
$$

Definition. For each $u \in \mathbf{N}$ we say that $d>u$ has property $\left(\mathbf{B}_{u}\right)$ if and only if

$$
\forall m \forall n\left(m<n=m+d \rightarrow \exists z_{1}, z_{2}, \ldots, z_{u}, m \prec z_{1} \prec z_{2} \prec \ldots \prec z_{u} \prec n\right) .
$$

Note. For all $d, d$ has property (A) if and only if $d$ has property $\left(\mathrm{A}_{1}\right)$ and if and only if $d$ has property $\left(\mathrm{B}_{1}\right)$. For all $k$ and for all $d, d$ has property $\left(\mathrm{B}_{k}\right)$ implies $d$ has property $\left(\mathrm{A}_{k}\right)$ which implies $d$ has property $\left(\mathrm{A}_{k+1}\right)$. For all $k$ and for all $d, d$ has property $\left(\mathrm{B}_{k}\right)$ implies $d+1$ has property $\left(\mathrm{B}_{k+1}\right)$, which implies $d+1$ has property $\left(\mathrm{A}_{k+1}\right)$.

It follows from the above note that if Conjecture 1 is true then all $d>1$ have property $\left(\mathrm{A}_{2}\right)$. It will follow from Theorem 5 and Corollary 8 that if Conjecture 1 is true then all $d>2$ have property $\left(\mathrm{B}_{2}\right)$.

We now gather further evidence to suggest that all $d>2$ have property $\left(B_{2}\right)$, in turn providing even stronger evidence that all $d>1$ have property $\left(A_{2}\right)$.

Our next result is based on Theorem 4.

THeOREM 5. Let $d_{1}$ have property (A). If $p$ is a prime such that $p \nmid d_{1}$ and $k \geq 0$, then $d_{2}=d_{1}+p^{k}$ has property $\left(\mathrm{B}_{2}\right)$.

Proof. Consider $m$ with $m<z_{1}<m+d_{1}$ illustrating property (A). If $p \mid m$ we have $m<z_{1}<z_{2}=m+d_{1}<n=z_{2}+p^{k}$. If $p+m$ we have $m<m+p^{k}=z_{1}<z_{2}<n=z_{1}+d_{1}$. 
COROLLARY 6. If $q_{1}$ and $q_{2}$ are primes (not necessarily distinct), then $d_{2}=$ $q_{1}+q_{2}+1$ has property $\left(\mathbf{B}_{2}\right)$.

Proof. Case 1. $q_{1}+q_{2}=5$ and so $d_{2}=6$. If $2 \mid m$ and $2 \mid n$ then $m<z_{1}=$ $m+1<z_{2}=m+5<n$ does the job. If $2+m n$, then $m<z_{1}=m+2<$ $z_{2}=m+4<n$ does the job.

Case 2. $q_{1}+q_{2} \neq 5$. Without loss of generality, suppose $q_{1} \geq q_{2}$. Then $q_{1} \nmid q_{2}+1$. By Theorem $4, d_{1}=q_{2}+1$ has property (A). So, by Theorem 5 , $d_{2}=q_{1}+q_{2}+1$ has property $\left(\mathbf{B}_{2}\right)$.

COROLlary 7. If Goldbach's conjecture is true, then all odd $d_{2} \geq 3$ have property $\left(\mathrm{B}_{2}\right)$.

COROLlaRY 8. If $d_{1}$ is odd and has property (A), and $k \geq 1$, then $d_{2}=$ $d_{1}+2^{k}$ has property $\left(\mathrm{B}_{2}\right)$.

These results tend to suggest that all odd $d \geq 3$ have property $\left(\mathbf{B}_{2}\right)$. (This would in turn imply that all $d>1$ have property $\left(\mathrm{A}_{3}\right)$.) Evidence that all even $d \geq 4$ have property $\left(B_{2}\right)$ follows again from Theorem 5 requiring $d_{1}$ and $p$ to be odd (and possibly $k$ to be zero).

Having gathered our evidence, we finish with two conjectures.

CONJECTURE 2. All $d \geq 3$ have property $\left(\mathrm{B}_{2}\right)$.

CONJECTURE 3. All $d \geq 2$ have property $\left(\mathrm{A}_{2}\right)$.

We recall that Conjecture 1 implies Conjecture 2, which implies Conjecture 3.

\section{Note added in proof}

The author has written a computer program whose output to date tells us that Conjecture 1 holds for $1 \leq m<n \leq 3,000,000$. Furthermore, the output tells us that the only value of $d$ shown not to have property (A) from inspecting $1 \leq m<n \leq 3,000,000$ is $d=16$.

Recalling the note after Conjecture 1 , for a given $d$ let $\pi(d)$ equal the product of all primes less than $d$. We note that if $d$ does not have property (A) and if the relevant (counter-)example $(m, n)$ has each prime less than $d$ either dividing $m$ or dividing $n$, then clearly $\pi(d) \mid m n=m(m+d)$ and so $m>\sqrt{\pi(d)}-d / 2$. Now, since $\pi(53)>5,000,000,053^{2}$ and since Conjecture 
1 holds for all odd $d \leq 89$, the evidence that Conjecture 1 likewise holds for $1 \leq m<n \leq 5,000,000,000$ is overwhelming.

We conclude that the approach of sequentially checking $m$ and $n$ (as in the author's program) is sluggish in the extreme compared to the alternative approach of checking each value of $d$ in turn; although the latter would undoubtedly constitute a more difficult programming exercise. A copy of the author's program (written in Pascal), which sequentially checks $m$ and $n$, is available from the author upon request.

\section{Acknowledgements}

I thank Alan Woods for comments regarding the presentation of this paper, and I thank the anonymous referee who checked further cases in support of Conjecture 1. I also thank Professor R. C. Vaughan for directing me to the result in [2] (and also for providing, in a private communication, an independent proof of Corollary 3). Finally, I would like to thank Dr Rod Worley of Monash University for his interest and for originally showing the result of Corollary 3 to Professor Vaughan.

An earlier version of this paper appears in the author's Ph.D. thesis [1].

\section{References}

[1] D. L. Dowe, Some aspects of program verification and program inversion, ( $\mathrm{Ph} . \mathrm{D}$. thesis, Monash University, Australia, 1985-86).

[2] $Y$. Motohashi, 'A note on the least prime in an arithmetic progression with a prime difference', Acta Arith. 17 (1970), 283-285.

[3] A. R. Woods, Some problems in logic and number theory, and their connections, (Ph.D. thesis, University of Manchester, 1981).

Department of Mathematics

Monash University

Clayton, Victoria 3168

Australia 